\title{
Generalized Hohenberg-Kohn theorem and effective Hamiltonian of a quantum system
}

\author{
T. K. Ng
}

AT\& T Bell Laboratories, Murray Hill, New Jersey 07974

(Received 1 February 1991; revised manuscript received 13 May 1991)

\begin{abstract}
It is proved that, for quantum systems with a nondegenerate ground state, there exists a one-to-one mapping in the Hohenberg-Kohn sense between any subset of the sets of parameters (functions) characterizing the Hamiltonian and a corresponding set of correlation functions that characterize the ground state of the system. Using this result, we study the problem of constructing an effective Hamiltonian out of known ground-state properties of a quantum system.
\end{abstract}

Recently, there has been some interest in the construction of effective Hamiltonians out of known ground-state properties of quantum systems, most noticeably in the construction of effective three-band or one-band models out of the local-density approximation (LDA) calculated ground-state properties of the high- $T_{c}$ copper oxide systems. $^{1-3}$ These works are based on physically motivated methods and so far, a rigorous mathematical justification for these works has not been available.

The purpose of this paper is to partially fill up this gap by proving a theorem which enables one to extract the exact Hamiltonian of a quantum system from a set of its ground-state correlation functions. We shall also discuss the implications of the theorem on the approximate methods in which the quantum Hamiltonians are extracted at present from LDA calculations. ${ }^{2-4}$

Consider a quantum system characterized by the Hamiltonian

$$
\begin{aligned}
& H=H_{1}+H_{2}, \\
& H_{1}=\sum_{i} A_{i} \hat{O}_{i}
\end{aligned}
$$

and

$$
H_{2}=\sum_{i} B_{i} \hat{P}_{i}
$$

where $\{A\}$ and $\{B\}$ are sets of parameters (functions) characterizing the Hamiltonian, and $\hat{O}_{i}$ and $\hat{P}_{i}$ are Hermitian operators operating on the Hibert space of the quantum system. The division of the Hamiltonian into two parts $H_{1}$ and $H_{2}$ is completely arbitrary. We shall prove the following theorem: Suppose all the parameters $A_{i}$ 's and thus $H_{1}$ is known; then there exists a one-to-one mapping between the set of parameters $\left\{B_{i}\right\}$ characterizing $\boldsymbol{H}_{2}$ and the set of ground-state correlation functions $\left\{\boldsymbol{P}_{i}\right.$ $\left.=\left\langle G\left|\hat{P}_{i}\right| G\right\rangle\right\}$ provided that the ground state is unique (nondegenerate).

The proof of this theorem is a straightforward extension of the proof of the Hohenberg-Kohn theorem. ${ }^{5}$ First of all, provided the ground state is unique, the set of parameters $\left\{B_{i}\right\}$ always determines uniquely the set of correlation functions $\left\{\boldsymbol{P}_{i}\right\}$. To prove that the converse is true, we assume that there exists two sets of parameters $\left\{B_{i}{ }^{(1)}\right\}$ and $\left\{B_{i}{ }^{(2)}\right\}$ which gives the same set of correlation functions $\left\{P_{i}\right\}$. Then from the principle of minimization of energy, we have

$$
\left\langle G^{(1)}\left|H^{(1)}\right| G^{(1)}\right\rangle<\left\langle G^{(2)}\left|H^{(1)}\right| G^{(2)}\right\rangle,
$$

where $\left|G^{(i)}\right\rangle$ is the ground-state wave function associated with the set of parameters $\left\{B^{(i)}\right\}$. Writing

$$
\left\langle G^{(2)}\left|H^{(1)}\right| G^{(2)}\right\rangle=\left\langle G^{(2)}\left|H^{(2)}+\left(H^{(1)}-H^{(2)}\right)\right| G^{(2)}\right\rangle
$$

we find

$$
E_{G}^{(1)}\left\langle E_{G}^{(2)}+\sum_{i}\left(B_{i}^{(1)}-B_{i}^{(2)}\right) P_{i} .\right.
$$

Notice that $P_{i}^{(1)}=P_{i}^{(2)} \forall i$ by assumption and $E_{G}^{(i)}$ is the ground-state energy of $H^{(i)}$. Similarly, one can prove that

$$
E_{G}^{(2)}\left\langle E_{G}^{(1)}+\sum_{i}\left(B_{i}^{(2)}-B_{i}^{(1)}\right) P_{i}\right.
$$

so that $E_{G}^{(1)}+E_{G}^{(2)}<E_{G}^{(1)}+E_{G}^{(2)}$ by adding (3a) and (3b). This is certainly impossible, thus proving our theorem. Notice that in the case $H_{1}=0$ so that $H_{2}$ is the whole Hamiltonian, the set of parameters $\left\{B_{i}\right\}$ is determined by the set $\left\{\boldsymbol{P}_{i}\right\}$ only up to an overall multiplicative factor, since two Hamiltonians differing only by an overall multiplicative factor gives the same ground-state wave function $|G\rangle$.

To understand the content of this theorem, let us consider a Hamiltonian for a system of interacting particles

$$
\begin{aligned}
H= & \frac{-1}{2 m} \sum_{i} \hat{\psi}^{\dagger}\left(\mathbf{r}_{i}\right) \nabla^{2} \hat{\psi}\left(\mathbf{r}_{i}\right)+\sum_{i} v\left(\mathbf{r}_{i}\right) \hat{n}\left(\mathbf{r}_{i}\right) \\
& +\sum_{i j} \phi\left(\mathbf{r}_{i}, \mathbf{r}_{j}\right) \hat{n}\left(\mathbf{r}_{i}\right) \hat{n}\left(\mathbf{r}_{j}\right),
\end{aligned}
$$

where $\hat{n}(\mathbf{r})=\hat{\psi}^{\dagger}(\mathbf{r}) \hat{\psi}(\mathbf{r})$ is the density operator at site $\mathbf{r}$. Suppose that the mass $m$ and the interaction potential $\phi$ is known, then our theorem states that there exists a one-toone mapping between the external potential $v(\mathbf{r})$ and the ground-state density distribution function $n(r)$ if the ground state is unique, i.e., the usual Hohenberg-Kohn (HK) theorem. However, our theorem also implies, for example, that if the mass $m$ and the external potential $v(r)$ is known, then the interaction potential completely determines and is completely determined by the groundstate pair-correlation function $\left\langle n\left(\mathbf{r}_{i}\right) n\left(\mathbf{r}_{j}\right)\right\rangle$. Lastly, the theorem also implies that if the ground-state correlation 
functions $\langle n\rangle,\langle n n\rangle$, and $\left\langle\psi^{\dagger} \nabla^{2} \psi\right\rangle$ are all known, then all the parameters specifying the Hamiltonian are determined up to an overall multiplicative factor, provided that the ground state is nondegenerate.

It is important to point out that since the theorem is just a direct extension of the Hohenberg-Kohn theorem, it suffers from all the deficiencies of the HK theorem as well. In particular, there are two outstanding problems. The first is proof of the theorem relies on the assumption that two different Hamiltonians $H^{(1)}$ and $H^{(2)}$ always give different ground-state wave functions, which is not true in general. For example, in the Hamiltonian (4), one can always add a constant potential to the Hamiltonian without changing the ground-state wave function, and the resulting ground-state correlation functions will not be modified. More generally, one can always add an extra term to the Hamiltonian which has no effect on the states in the Hibert space of interest and thus has no effect on the ground state of the system. However, these terms are usually trivial and can be excluded from the theorem quite easily. ${ }^{5}$ A more nontrivial situation which may occur is that two Hamiltonians differing in a nontrivial way may have the same ground-state wave function, as was demonstrated by von Barth and Hedin ${ }^{6}$ in the case of a single electron moving in a magnetic field. ${ }^{6}$ The generalized Hohenberg-Kohn (GHK) theorem is not applicable in this situation. However, this problem can be solved by going to finite temperature where one can prove a finite temperature generalization of the GHK theorem in a way parallel to Mermin's ${ }^{7}$ extension of the HK theorem to finite temperature. In the finite-temperature theorem, the ground-state wave function $|G\rangle$ is replaced by the grand canonical density matrix $\hat{\rho}=e^{-\beta(\hat{H}-\mu \hat{N})}$ and energy $E_{G}$ is replaced by the grand potential $\Omega=-1 / \beta \ln \operatorname{Tr} \hat{\rho}$. A oneto-one correspondence between the set of parameters $\left\{B_{i}\right\}$ and the set of equal-time correlation functions $\left\{\boldsymbol{P}_{i}\right\}$ can then be proved in a way parallel to the proof of the (ground state) GHK theorem, with, however, a crucial difference. In the proof of the ground-state GHK theorem, it has to be assumed that two different Hamiltonians $\boldsymbol{H}^{(1)}$ and $\boldsymbol{H}^{(2)}$ always give different ground-state wave functions, which is not always true. However, in the finite-temperature case, the corresponding quantity $\hat{\rho}=e^{-\beta(\hat{H}-\mu \hat{N})}$ is always different for different Hamiltonians (unless the differences are trivial ones as discussed above). Thus the same ground-state wave function problem in the zero-temperature theorem is not present in the finite temperature generalization.

The second problem which the GHK theorem suffers is that the theorem is in general noninvertible, i.e., there may exist sets of correlation functions $\left\{\boldsymbol{P}_{i}\right\}$ which do not correspond to ground-state wave functions of any Hamiltonian $\mathrm{H}_{2}$ (Ref. 8) ( $v$ representability problem). This problem is not solvable just by going to finite temperature and is important if one wants to formulate a variational procedure to find the ground-state wave function of a given Hamiltonian by minimizing the energy with respect to the set of correlation function $\left\{P_{i}\right\}$ (i.e., formulating Kohn-Sham-type equations ${ }^{9}$ ). In this case, the problem can be solved by an approach parallel to Levy ${ }^{8}$ where one considers only the sets of correlation function $\left\{\boldsymbol{P}_{i}\right\}$ which are $N$ representative, i.e., it is the correlation function of some existing wave function $\psi_{N}$, and the variational procedure is carried out only in this restricted set of correlation functions. ${ }^{8}$ We shall not go into detail here since the $v$ representability problem is not crucial to our forthcoming discussions.

The consequences of this theorem are rich, as can be seen from the above discussion. We shall confine ourselves to just one aspect in the following, that any quantum Hamiltonian $H=\Sigma_{i} B_{i} \hat{P}_{i}$ is determined (i.e., the set of parameters $\left\{B_{i}\right\}$ is specified) by the knowledge of the set of correlation functions $\left\{\boldsymbol{P}_{i}\right\}$, up to an overall multiplicative factor, provided that the ground state is nondegenerate (or the corresponding finite-temperature statement).

Suppose we want to construct an effective Hamiltonian $H=\sum_{i} B_{i} \hat{P}_{i}$ describing only part of the dynamics of a complicated realistic system, then the above theorem immediately implies that the set of parameters $\left\{B_{i}\right\}$ is uniquely determined (assuming that the ground state is nondegenerate) up to an overall multiplicative factor once we obtain the set of correlation functions $\left\{\boldsymbol{P}_{i}\right\}$ from the original physical system. Notice that the theorem does not specify the way in which the set of correlation functions $\left\{\boldsymbol{P}_{i}\right\}$ should be extracted from the original system, it just states which correlation functions one should extract from the original physical theorem in order to completely determine the effective Hamiltonian. The physical reason for this is simple: the construction of an effective Hamiltonian from a complicated physical system amounts to projecting the dynamics of the complete phase space of the physical system onto a smaller subspace of interest. The way of defining the projected subspace is not unique. It depends on the way of defining the projection process and so does the parameters specifying the effective Hamiltonian.

However, the theorem does suggest a test for the correctness of $H_{\text {eff }}$ in describing the underlying physical system, by comparing various ground-state correlation functions of the real and model systems. In particular, if the difference between a particular correlation function is large, then our theorem suggests that a corresponding term should be added to the effective Hamiltonian in order to model the real system more accurately.

A recent topic of interest is the construction of a threeband model from the knowledge of the ground-state properties of the high- $T_{c}$ copper oxide systems from LDA band-structure calculations. ${ }^{2,3}$ We shall comment on what our theorem tells about these calculations in the following.

The three-band model of interest is a generalized Hubbard model of the form

$$
\sum_{\langle i j\rangle} \epsilon_{i j} C_{i \sigma}^{\dagger} C_{j \sigma}+\sum_{\langle i j\rangle_{\sigma \sigma^{\prime}}} U_{i j} C_{i \sigma}^{\dagger} C_{i \sigma} C_{j \sigma^{\prime}}^{\dagger} C_{j \sigma^{\prime}}
$$

where $C$ 's are usual electron operators and $\langle i, j\rangle$ denote both site and band indices. From our existence theorem we know that the parameters $\epsilon_{i j}$ 's and $U_{i j}$ are all uniquely determined by the set of correlation functions $\left\langle C_{i \sigma}^{\dagger} C_{j \sigma}\right\rangle$ and $\left\langle n_{i \sigma} n_{j \sigma}\right\rangle$. These correlation functions can, in principle, be extracted from a LDA density functional calculation ${ }^{10}$ and the values of $\epsilon_{i j}$ 's and $U_{i j}$ 's can be obtained approxi- 
mately by comparing these numbers with numbers obtained from approximate solutions of the three-band model (e.g., by Hartree-Fock or random-phase approximation).

In practice, however, although the kinetic energy terms $\left\langle C_{i \sigma}^{\dagger} C_{j \sigma}\right\rangle$ can be obtained quite straightforwardly from LDA calculation, the equal-time correlation functions $\left\langle n_{i \sigma} n_{j \sigma}\right\rangle$ are more difficult to obtain ${ }^{10,11}$ and the present calculations are all performed by effectively comparing instead the zero frequency correlation functions $\left\langle n_{i \sigma}\right.$ $\left.\times n_{j \sigma^{\prime}}\right\rangle_{\omega}=0$, which can be obtained in a LDA calculation by simply putting on a static weak potential and looking at the (linear) response of the system. ${ }^{2,12}$ The zerofrequency response functions are then compared with corresponding results obtained from an approximate solution of the three-band model. In practice, these procedures might give very good estimates of the various parameters in the three-band model, but from the existence theorem point of view, these procedures are not warranted to give a unique effective Hamiltonian since the existence theorem relates only equal-time correlation functions to the parameters characterizing the Hamiltonian (unless, of course, an existence theorem relating zero-frequency correlation functions and the parameters characterizing the Hamiltonian can be proved). It would be interesting to see whether a computation with the "correct" correlation functions would give significantly different results from the present calculations. An alternative way of interpreting the results from the zero-frequency calculation is to consider an effective Hamiltonian of form (5), but with an additional cutoff in energy $\omega_{c}$ such that $\omega_{c} \ll E_{F}$ where $E_{F}$ is approximately the characteristic energy scales of $H_{\text {eff }}$ without cutoff. The parameters in $H_{\text {eff }}$ extracted from the zero-frequency correlation function can be viewed as the parameters appropriate for $H_{\text {eff }}$ with the cutoff $\omega_{c} \rightarrow 0$ (such that the equal-time correlation function becomes "averaged" over time scale $\tau \sim \omega_{c}^{-1}$ because of the uncertainty principle). Notice, however, that although the evaluation of the equal-time correlation functions are difficult in the LDA calculation, they are obtained naturally in exact diagonalization computations. Thus the present theorem provides a natural guidance for construction of, say, effective one-band Hubbard models from given three-band models, where exact diagonalization for small clusters can be carried out in both cases. ${ }^{2}$ The parameters for the one-band model can be fixed by comparing the equal-time correlation functions of the two models.

In conclusion, we have proven an existence theorem which is a generalization of the Hohenberg-Kohn theorem to a quantum system with arbitrary Hamiltonian. The implications of this theorem are rich and we have not yet explored all possible consequences of the theorem. (For example, the theorem includes the Hohenberg-Kohn theorem $^{5}$ and its generalization to the case with static magnetic fields ${ }^{13}$ as special cases and it has also been used to study the metal-insulator behavior of certain wave functions on lattice models. ${ }^{14}$ ) We have, however, applied the theorem to study the problem of construction of effective Hamiltonian from known ground-state properties of a quantum system and point out that our theorem offers a qualitative guidance for the construction of the effective Hamiltonian. We point out also that the present procedures of constructing multiband models out of a LDA band-structure calculation of high- $T_{c}$ copper oxide systems is not warranted to give the correct effective Hamiltonian, from the point of view of our existence theorem.

The author thanks M. S. Hybertsen and A. J. Millis for useful discussions.
' J. G. Bednorz and K. A. Müller, Z. Phys. B 64, 188 (1986).

${ }^{2}$ M. S. Hybertsen, M. Schluter, and N. E. Christensen, Phys. Rev. B 39, 9028 (1989).

${ }^{3}$ A. K. McMahan, R. M. Martin, and S. Satpathy, Phys. Rev. B 38, 6650 (1989).

${ }^{4}$ O. Gunnarsson, O. K. Anderson, O. Jepsen, and J. Zaanen, in Proceedings of the Tenth Tanigashi Symposium on Core Level Spectroscopics (Springer, Berlin, 1989).

${ }^{5}$ P. Hohenberg and W. Kohn, Phys. Rev. 136, B864 (1964).

${ }^{6}$ U. von Barth and L. Hedin, J. Phys. C 5, 1629 (1972).

${ }^{7}$ N. D. Mermin, Phys. Rev. 137, A1441 (1965).

${ }^{8}$ M. Levy, Proc. Natl. Acad. Sci. U.S.A. 76, 6062 (1979).
${ }^{9}$ W. Kohn and L. J. Sham, Phys. Rev. 140, A1133 (1965).

${ }^{10}$ The frequency-dependent density-density response function can be computed via density-functional theory (Ref. 11) and the equal-time correlation function $\left\langle n(\mathbf{r}) n\left(\mathbf{r}^{\prime}\right)\right\rangle$ can be obtained via fluctuation dissipation theorem.

${ }^{11}$ T. K. Ng, Phys. Rev. B 40, 8061 (1989); E. K. U. Gross and W. Kohn, Phys. Rev. Lett. 55, 2850 (1985).

${ }^{12}$ P. H. Dederichs, S. Blugel, R. Zeller, and H. Akai, Phys. Rev. Lett. 53, 2512 (1984).

${ }^{13}$ G. Vignale and M. Rasolt, Phys. Rev. B 37, 10685 (1988).

${ }^{14}$ A. J. Millis and S. N. Coppersmith, Phys. Rev. B 43, 13770 (1991). 